\title{
THE COARSE STRUCTURE OF THE SOLAR ATMOSPHERE*
}

\author{
M. SIMON' and H. ZIRIN \\ Mount Wilson and Palomar Observatories, Carnegie Institution of Washington, \\ California Institute of Technology, Pasadena, Calif., U.S.A.
}

(Received 19 May, 1969)

\begin{abstract}
Observations of the quiet sun at wavelengths from $3 \AA$ to $75 \mathrm{~cm}$ show (with two exce ptions: the Ovi line at $1032 \AA$ and possibly the continuum at $1.2 \mathrm{~mm}$ ) either no limb brightening or less than had been supposed. On the other hand, the brightness temperature is observed to increase with wavelength in the millimeter and centimeter range. If this increase is due to greater visibility of hot overlying material, that material ought to be evident at the limb at shorter wavelengths, resulting in limb brightening. The only possible explanation for the absence of limb brightening at almost all wavelengths is that the emitting surface is rough at all wavelengths, with a scale of roughness approximately equal to the scale height at each temperature. Contradictions with existing models, along with the additional observations required for an improved model are discussed.
\end{abstract}

\section{Introduction}

For many years astronomers have sought to detect the upward increase in temperature in the solar atmosphere by limb brightening at some wavelengths. It was argued logically that the increase in path length near the limb would enable us to see higher and hotter regions near the limb. This behavior was to be expected in the ultraviolet and the radio range. Particularly in the radio range, the central brightness temperature was known to increase with wavelength; hence it was expected (because the opacity depends only on $\lambda^{2} \sec \theta$ in a spherically symmetric model) that observations near the limb at one wavelength would show the increased temperature observed at the next higher wavelength.

The radio observations which were available up to 1965 were summarized by Kundu (1965) and most of the quiet sun data did in fact show limb brightening. However, new data shows that there is less, if any, limb brightening than had been supposed from older observations, and also that there is much less than is predicted by available models of the solar atmosphere. Only two observations at the short wavelengths in the radio range show any brightening near the limb: The famous work of Coates et al. (1958) which ruled out a secant-like brightening at $8.6 \mathrm{~mm}$ but indicated a possible spike near the limb; and the data of Noyes et al. (1968) and Newstead (1969) who found some evidence for limb brightening at $1.2 \mathrm{~mm}$, but could not eliminate the effect of plages and active regions. Lena (1968) at $25 \mu$, Shimabukuro and Stacey (1968) at $3.4 \mathrm{~mm}$, Tlamicha (1968) at $2 \mathrm{~cm}$ and Moffet (1967) at $3 \mathrm{~cm}$ all found little or no evidence of limb brightening. The recent observations are summarized in Table $\mathrm{I}$. Where there is disagreement with older observations, the reason appears to be that improved ob-

* This research was supported by the National Science Foundation under GP-7030 and the National Aeronautics and Space Administration under grant NGR 05002034.

+ Present address: Dept. of Earth and Space Sciences, State University of New York, Stony Brook, N.Y., U.S.A.

Solar Physics 9 (1969) 317-327; (C) D. Reidel Publishing Company, Dordrecht-Holland 
TABLE I

Summary of recent observations of limb distribution $\left(\varrho=r / R_{\odot}\right)$

\begin{tabular}{|c|c|c|c|}
\hline Wavelength & Reference & Results & Comments \\
\hline $10 \mu$ & Lena (1968) & Limb darkening & \\
\hline $25 \mu$ & Lena (1968) & Flat distribution & \\
\hline $1.2 \mathrm{~mm}$ & Noyes et al. (1968) & $\begin{array}{l}\text { Some limb brightening at } \\
\text { the equator }\end{array}$ & $\begin{array}{l}\text { Possibly in agreement with } \\
\text { BCA model }\end{array}$ \\
\hline $1.2 \mathrm{~mm}$ & Newstead (1969) & Some limb brightening & \\
\hline $3.4 \mathrm{~mm}$ & $\begin{array}{l}\text { Shimabukuro and } \\
\text { Stacey (1968) }\end{array}$ & Flat to $\varrho \geqslant 0.7$ & $\begin{array}{l}\text { In conflict with all recent } \\
\text { models }\end{array}$ \\
\hline $8.6 \mathrm{~mm}$ & Coates (1958) & $\begin{array}{l}\text { Flat, with possible spike } \\
\text { at limb }\end{array}$ & $\begin{array}{l}\text { In conflict with all recent } \\
\text { models }\end{array}$ \\
\hline $2 \mathrm{~cm}$ & Tlamicha (1968) & Flat to $\varrho \geqslant 0.8$ & $\begin{array}{l}\text { In conflict with all recent } \\
\text { models }\end{array}$ \\
\hline $3 \mathrm{~cm}$ & Moffet (1967) & $\sim$ Flat to $\varrho \geqslant 0.8$ & $\begin{array}{l}\text { In conflict with all recent } \\
\text { models }\end{array}$ \\
\hline $9 \mathrm{~cm}$ & Riddle (1969) & $\begin{array}{l}\text { Flatter than older results; } \\
\text { limb brightening at } \\
\text { equator only }\end{array}$ & $\begin{array}{l}\text { In conflict with all recent } \\
\text { models }\end{array}$ \\
\hline $75 \mathrm{~cm}$ & Little (1968) & $\begin{array}{l}\text { Flatter than older results; } \\
\text { limb brightening at } \\
\text { equator only }\end{array}$ & $\begin{array}{l}\text { In conflict with all recent } \\
\text { models }\end{array}$ \\
\hline
\end{tabular}

servations at the shorter wavelengths are now possible with instruments of higher resolution. Since active regions are not fully resolved in low resolution observations, foreshortening produces a spurious increase near the equatorial limb. For this reason some observations show equatorial limb brightening but no polar limb brightening. We cannot overemphasize the necessity of eliminating active regions from the radio $b^{1 y^{h}}$ data; ideally north-south scans are required. Obviously the coronal contribution will be small at the poles, but the chromosphere should be spherically symmetric, and polar data offers the best way to study the quiet chromosphere.

We shall concentrate our discussion in the radio data. However, the same regions that are responsible for the centimeter and decimeter radio emission are also observable in completely different wavelength regions and it is very interesting to survey those results. Bonnet and Blamont (1968) found no limb brightening between 1800 and $2900 \AA$. Tousey and Purcell (Tousey, 1967) obtained a series of beautiful spectroheliograms in the extreme ultraviolet resonance lines. Although a spike at the limb was found in a few lines (such as NevII, Ov, and Fex-XIV coronal lines) no secant-like brightening was observed in any line. The only secant-like brightening yet observed was found by Noyes and Goldberg for OVI at $1032 \AA$. The Harvard experimenters have not published any other lines that show limb brightening; their spectroheliogram in $\mathrm{Mgx} 625 \AA$ shows only a number of active regions near the limb, and the Lyman 
continuum shows limb darkening. Similarly, Vaiana et al. (1968) obtained high resolution pictures between 3.5 and $50 \AA$ which also show no limb brightening even though a number of small plages are detected.

Thus a model of the solar atmosphere is required to be in consonance with the absence of limb brightening at all wavelengths except $1032 \AA$, even though the temperature must increase outward at some point between the top of the photosphere $\left(4500^{\circ}\right)$ and the corona $\left(1000000^{\circ}\right)$. The model may include a limb spike which is undetectable in many of these observations which otherwise rule out secant-like limb brightening.

\section{The Radio Data}

The radio emission of the quiet sun is thermal free-free emission of the solar atmosphere. At meter wavelengths, the emission observed is that of the corona, and the deepest level observed is set by the local plasma frequency. At millimeter and centimeter wavelengths the optical depth becomes $\sim 1$ in the chromosphere and well above the regions where the plasma frequency would become important. Thus the main emphasis in the next section will be on straightforward solutions of the equation of radiative transfer. We describe the observations in terms of the brightness temperature $T_{b}$ which is calculated from the observed brightness by use of the Rayleigh-Jeans approximation. The fundamental data are the distribution of brightness temperature $T_{b}(\varrho, \lambda)$ in both wavelength $\lambda$ and position $\varrho$ on the quiet solar disk, where $\varrho=r / R_{\odot}=\sin \theta, \theta$ being measured from the center of the disk. We shall discuss the central brightness temperatures $T_{b}(0, \lambda)$ and the normalized center-to-limb distributions $T_{b}(\varrho, \lambda) / T_{b}(0, \lambda)$ separately.

The available $T_{b}(0, \lambda)$ have been recently compiled by Shimabukuro and Stacey (1968). There is scatter among the many observations, and we have indicated the range of the observations at millimeter and centimeter wavelengths by the shaded swath in Figure 1. It is necessary to know the actual distribution to better accuracy than that represented in the figure; we thus comment in the probable sources of the scatter in the observations and attempt to infer the actual distribution. At centimeter wavelengths the scatter is probably due mostly to the difficulty in obtaining a truly 'quiet' sun value. Many of the observations reported in Shimabukuro and Stacey's compilation did not of course attempt specifically to represent a quiet sun point, and of those that did, many, particularly the older observations, are 'disk' temperatures and thus include an average over any active regions that were present. Even statistical extrapolation to zero sunspot number may lead to high values because of the persistence of plages even after the obvious optical active regions have disappeared. The quiet sun values thus obtained are invariably overestimates. In a recent measurement intended specifically to obtain the quiet sun brightness temperature at $2 \mathrm{~cm}$, Buhl and Tlamicha (1968) obtained $T_{b}(0,2 \mathrm{~cm})=9100 \pm 200 \mathrm{~K}$ which falls near the lower boundary of the swath in Figure 1. We may expect that the actual distribution $T_{b}(0, \lambda)$ does follow the lower bound in Figure 1 and clearly further narrow-beam observations of undisturbed regions on the sun are needed. At millimeter wavelengths on the other hand the effects of solar 


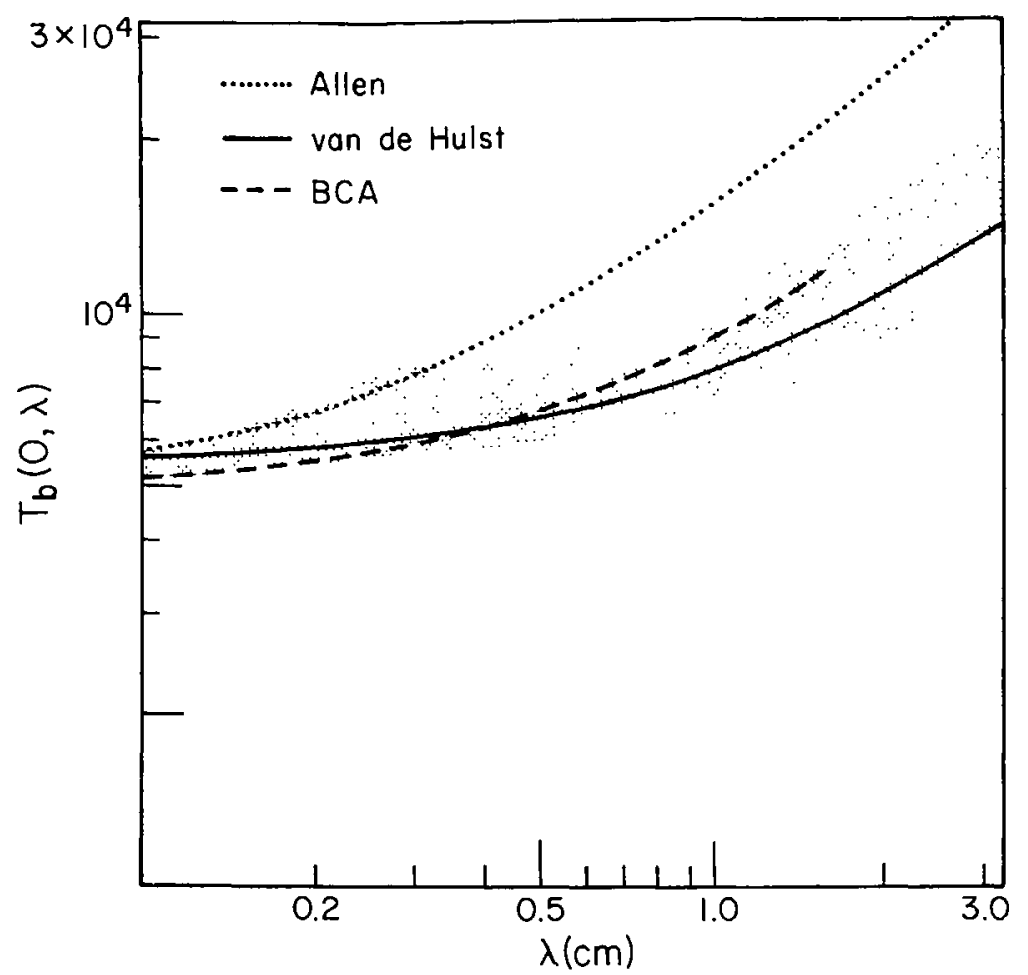

Fig. 1. The shaded region represents the range of measurements of the central solar brightness temperature as a function of wavelength (based in part on compilation of Shimabukuro and Stacey, 1968a). Theoretical central brightness temperature distributions for the Allen (1963), Van de Hulst

(1950), and BCA models (Gingerich and De Jager, 1968; Noyes et al., 1968) are indicated.

activity are less important; at $3.4 \mathrm{~mm}$ a typical plage contributes only about $200 \mathrm{~K}$ to the total brightness temperature (Simon, 1965). The scatter at these wavelengths is probably due to the large (and variable) contribution from the earth's atmosphere and the difficulties in obtaining absolute calibration.

We turn now to the center-to-limb brightness distributions and discuss the observations that have been carried out at $2 \mathrm{~cm}, 8.6 \mathrm{~mm}, 3.4 \mathrm{~mm}$, and $1.2 \mathrm{~mm}$. With the exception of the $8.6 \mathrm{~mm}$ observations, the data represents $T_{b}(\varrho, \lambda)$ convoluted with the

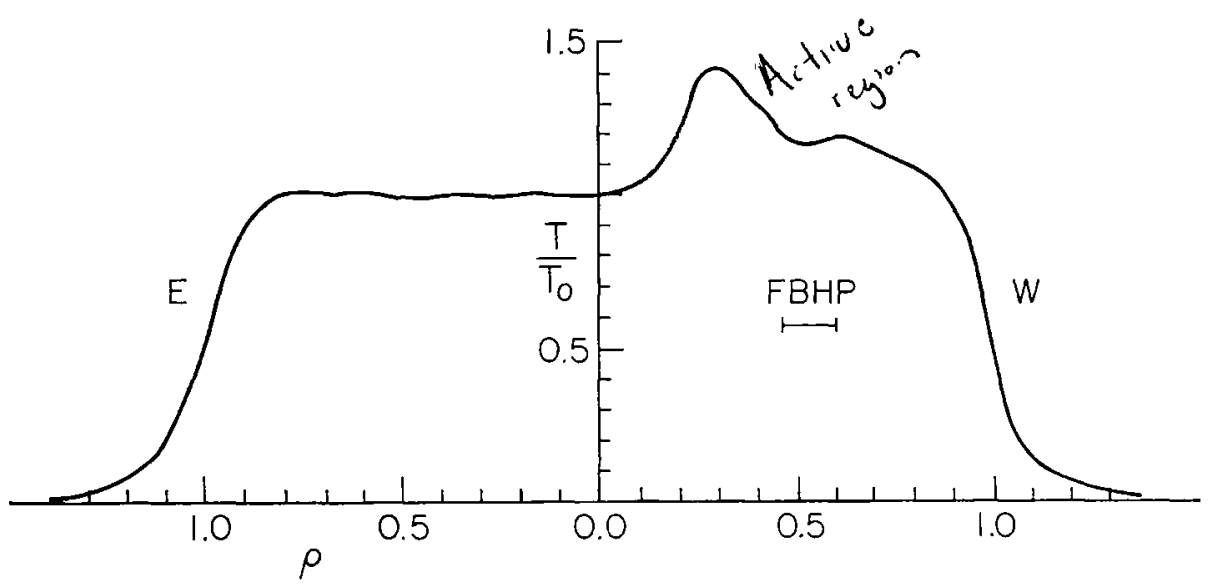

Fig. 2. East-West scan of the sun at $2 \mathrm{~cm}$ obtained by Tlamicha (1968) with the $140^{\prime}$ NRAO telescope. The width of the full beam at half power is indicated. 
antenna pattern. Tlamicha (1968) has recently obtained scans of the sun with the $140^{\prime}$ radio telescope of the NRAO ( $\sim 2$ min of arc beamwidth). We reproduce one of his scans in Figure 2 and direct attention to the easterns portion of the scan which had no active regions. Because of the convolution, it is impossible to infer the distribution near the limb; it is apparent nonetheless that $T_{b}(\varrho, 2 \mathrm{~cm}) \sim$ const. (to within $2 \%$ ) for $\varrho<0.82$. We show in Figure 3 a north-south scan across the sun at $3 \mathrm{~cm}$ obtained by

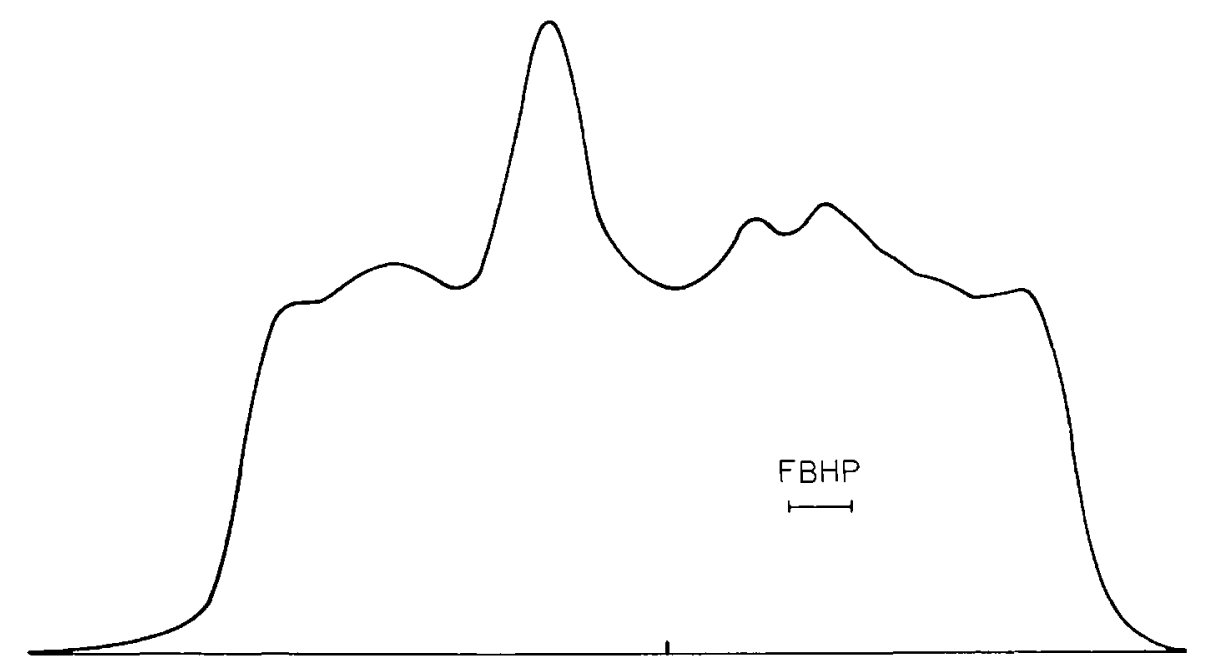

Fig. 3. North-South scan of the sun at $3 \mathrm{~cm}$ obtained by Moffet (1968) with the $210^{\prime}$ Goldstone telescope. The width of the full beam at half power is indicated.

Moffet (1967) with the Goldstone 210' antenna. Detailed analysis is unfeasible since the sun had a few large active regions at the time of the observations. It is clear nonetheless that the distribution near the limb is flatter than might have been expected from older observations at $3 \mathrm{~cm}$ (Alon et al., 1953). The results of the eclipse observations at $8.6 \mathrm{~mm}$ of Coates (1958) are well known; the high resolution possible in an eclipse showed that $T_{b}(\varrho, 8.6 \mathrm{~mm})$ was again essentially constant, with possibly a narrow spike at the limb. Shimabukuro and Stacy (1968) presented a distribution at 3.4 $\mathrm{mm}$ which is the average of three scans selected to represent the quiet sun. Since the observations are convoluted with the 2.8 min of arc beam of the Aerospace Corporation telescope, it is again impossible to discuss the distribution at the limb. Their average distribution shows a slight rise beyond $\varrho \sim 0.7$. It must be remembered however that the averaging of scans retains any spurious enhancements which might be attributable to small plages near the equator. The north-south distribution obtained by Shimabukuro (1969) shows no such enhancement. Noyes et al. (1968) carried out observations at $1.2 \mathrm{~mm}$. Although the beam pattern of their telescope was poorly known, they were able to infer the existence of limb brightening from the asymmetry of the intensity decrease at the east and west limb. From partial eclipse observations, Newstead (1969) found the ratio of the disk-averaged brightness temperature to that at the center of the disk to be $1.11 \pm 0.02$, confirming the existence of limb brightening. None of this work applied to the north-south limba where the effects of small plages would have been minimized. 
The general features that emerge from this discussion of the available data and that must be accounted for by a chromospheric model are the gradual rise in brightness temperatures from around $6000 \mathrm{~K}$ at $3 \mathrm{~mm}$ to about $9000 \mathrm{~K}$ at $2 \mathrm{~cm}$ and the remarkably flat brightness distribution across the disk at these wavelengths. As we discuss in the next section, it is the latter requirement that provides the severest test of chromospheric models.

The same analysis at longer wavelengths (where the corona contributes the bulk of the observed radio emission) shows that the structure of the lower corona is an extension of the same rough structure that we shall use for the chromosphere. Riddle (1969) has recently analyzed the $9.1 \mathrm{~cm}$ data obtained with the Stanford spectroheliograph during the quiet sun years 1964-5 and found considerably less enhancement at the limb than found from the 1960 data by Swarup (1961). Particularly, analysis of the north-south distribution, which would be least affected by active regions, showed no limb brightening. Significant also are the high resolution $75 \mathrm{~cm}$ observations with the Molonglo cross (Little, 1967). The center to limb brightness distribution again was observed to be flatter than expected.

\section{Application of Model Atmospheres to the Radio Data}

The brightness distribution $T_{b}(\varrho, \lambda)$ for a given solar atmosphere model is obtained by application of the equation of transfer. It has been suspected since Coates' (1958) and Athay's (1959) analyses of the $8.6 \mathrm{~mm}$ eclipse data that spherically symmetric homogenous models are in conflict with those observations. Because of their analytical convenience such models continue to be used and constructed. We shall thus first discuss the BCA (Gingerich and De Jager, 1968), Allen (1963), and Van de Hulst (1950) models and emphasize their inability to account for the observations in the wavelength range $3.4 \mathrm{~mm}<\lambda<2 \mathrm{~cm}$. Given the electron density and temperature distributions, the predicted brightness temperature is

$$
T_{b}(\varrho, \lambda)=\int_{0}^{\infty} T_{e}(h) \mathrm{e}^{-\tau(\varrho, \lambda, h)} \mathrm{d} h
$$

where the optical depth $\tau$ is

$$
\tau(\varrho, \lambda, h)=\frac{1}{\left(1-\rho^{2}\right)^{1 / 2}} \int_{0}^{\infty} K \mathrm{~d} h
$$

and the absorption coefficient $K$ is

$$
K=1.98 \times 10^{-23} g \frac{\lambda^{2} n_{e}^{2}}{\mu T^{3 / 2}} .
$$

The Gaunt factor $g$ varies slowly around $\sim 3$ in the chromosphere. For the wavelengths and models which we shall consider, the refractive index $\mu$ does not differ 
significantly from 1 in the regions that contribute to the emission. This is not true at longer wavelengths, and of course is not generally true for other models.

We show in Figure 1 the results of our calculation of $T_{b}(0, \lambda)$ for the Allen and Van de Hulst models. Noyes et al. (1968) have calculated $T_{b}(0, \lambda)$ for an extension of the BCA atmosphere; we have plotted their results in Figure 1 as well. In the sense that the distributions obtained from the Van de Hulst and BCA models fall in the swath in Figure 1, these models give better agreement with the data than the Allen model which consistently predicts higher temperatures. The extension of the BCA model to higher temperatures by Noyes, Beckers, and Low was however, specifically adjusted for agreement with the radio data.

We have calculated the center to limb brightness distribution to be expected on the basis of these models and present the normalized $2 \mathrm{~cm}$ and $3.4 \mathrm{~mm}$ results in Figure 4.
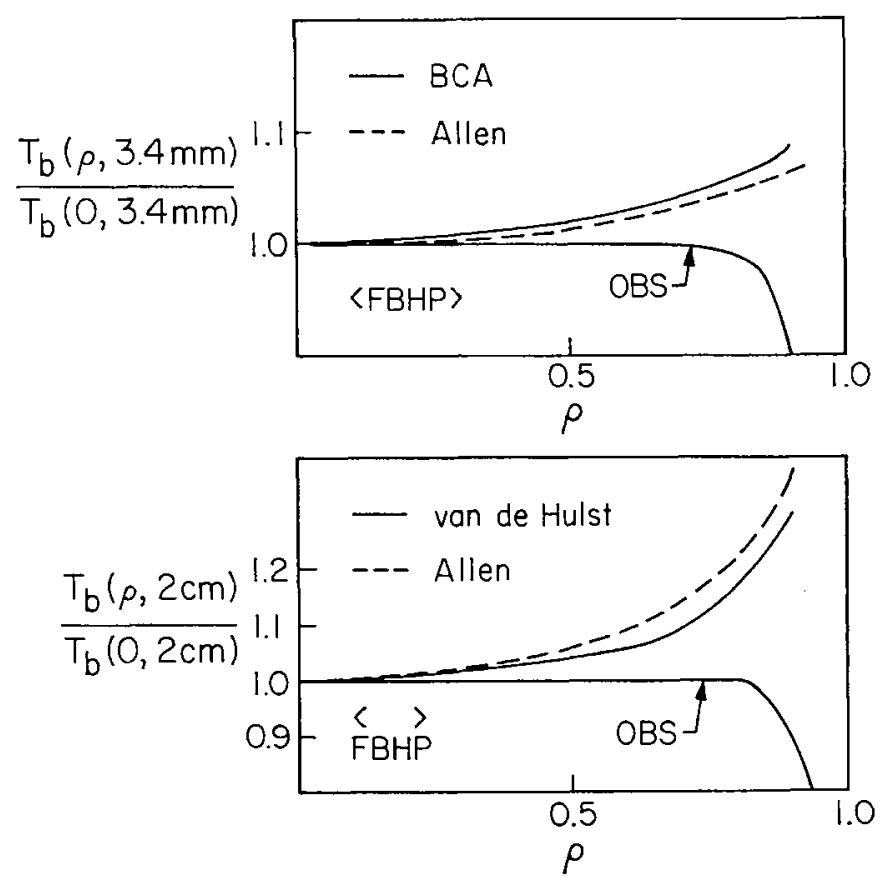

Fig. 4. Comparison of theoretical and observed distributions of brightness distributions across the limb. The observed $3.4 \mathrm{~mm}$ profile is an average north-south scan derived by Shimabukuro (1969), and the observed $2 \mathrm{~cm}$ profile is the eastern portion of Figure 2.

We have also reproduced in Figure 4 the $2 \mathrm{~cm}$ distribution observed by Tlamicha, and the north-south distribution at $3.4 \mathrm{~mm}$ reported by Shimabukuro (1969). Direct comparison between the observed and theoretical profiles is difficult because of the antenna convolution; we have indicated in the figure the half-power beam of the antenna used to obtain the data to show the degree of smoothing that is present in the data. It is obvious from even rough inspection of the figure that all the models predict far more limb brightening than is observed at $3.4 \mathrm{~mm}$ and $2 \mathrm{~cm}$. 


\section{Implications of the Radio Data}

A discontinuity in the brightness temperature is always to be expected at the solar limb since there the optically thin regions contribute from twice the volume. Neglecting that effect however, and the limb spike observed at $8.6 \mathrm{~mm}$ by Coates (1958) which was so large that it would have to be explained by an inhomogeneity such as spicules, the present observations are sufficient to exclude any simply spherically symmetric homogeneous model, including the 'hard sphere' models in which the optical depth decreases rapidly with height, proposed by Zirin and Dietz (1963). The reason is clear from (1) and (2) which show that $T_{b}(\varrho, \lambda)$ is a Laplace transform over $T_{e}(h)$ with the transform variable proportional to $\lambda^{2} \sec \theta$. We take as conservative working hypotheses that $T_{b}(\varrho, 2 \mathrm{~cm})$ is flat to at least $\varrho \sim 0.8$, that $T_{b}(\varrho, 3.4 \mathrm{~mm})$ and $T_{b}(\varrho, 8.6 \mathrm{~mm})$ are at least as flat and that $T_{b}(0, \lambda)$ in the centimeter range follows roughly along the lower bound of the swath in Figure 1. Then, independence of $T_{b}(\varrho, 2 \mathrm{~cm})$ of $\varrho$ for $0 \leqslant \varrho \leqslant 0.8$ (corresponding to $1 \leqslant \sec \theta \leqslant 1.6$ ), implies that $T_{b}(0, \lambda)$ should be independent of wavelength over wavelengths from $2 \mathrm{~cm}$ to $2.4 \mathrm{~cm}$, or using the millimeter data, from $8.6 \mathrm{~mm}$ to $1.1 \mathrm{~cm}$. Neither appears to be true, and it is evident that such a model simply cannot account for both the flat center to limb distributions, and the gradual increase in $T_{b}(0, \lambda)$ with increasing wavelength. A model such as the BCA does however give a satisfactory representation of the observations at $1.2 \mathrm{~mm}$ and less (where there is little observational data to test it). It is thus certain that the models must be revised in the chromospheric regions.

The observations at $9 \mathrm{~cm}, 21 \mathrm{~cm}$, and $75 \mathrm{~cm}$, all of which show flatter center to limb distributions than might have been expected on the basis of spherically symmetric homogeneous models indicate that models of the lower corona may also need to be revised. In fact the observations at $2 \mathrm{~cm}$ may already be indicating significant information about the lower corona. For simplicity, Equation 1 may be split into the chromospheric and coronal contributions. Since the corona is optically thin at millimeter and centimeter wavelengths, the coronal contribution is written,

$$
T_{\text {cor }}(\varrho, \lambda)=T_{\mathrm{c}} \tau(\varrho, \lambda)
$$

and if, as has been conventional, the corona is taken to be homogeneous and spherically symmetric, $\tau(\varrho, \lambda)=\tau_{0}\left(1-\varrho^{2}\right)^{-1 / 2}$. We have plotted $T_{\text {cor }}(0, \lambda)$ for Van de Hulst's minimum (1950) and Newkirk's quiet sun (1961) coronal models in Figure 4. It is seen that the flatness of the $2 \mathrm{~cm}$ observations is consistent with a homogeneous Van de Hulst model, but would be inconsistent with any homogeneous model that has a much higher $T_{\text {cor }}(0,2 \mathrm{~cm})$. If the brightness distribution is shown to be flat beyond $\varrho \sim 0.8$, then that will be inconsistent with even the homogeneous Van de Hulst coronal model, since that would give too much limb brightening.

It appears then that one is forced to drop the homogeneity assumption. Since all optical observations show complex structure, this is no surprise. The first attempt at a -nonhomogeneous model of the solar atmosphere is Athay's (1959) two component model, in which the relative volumes occupied by each component as a function of 


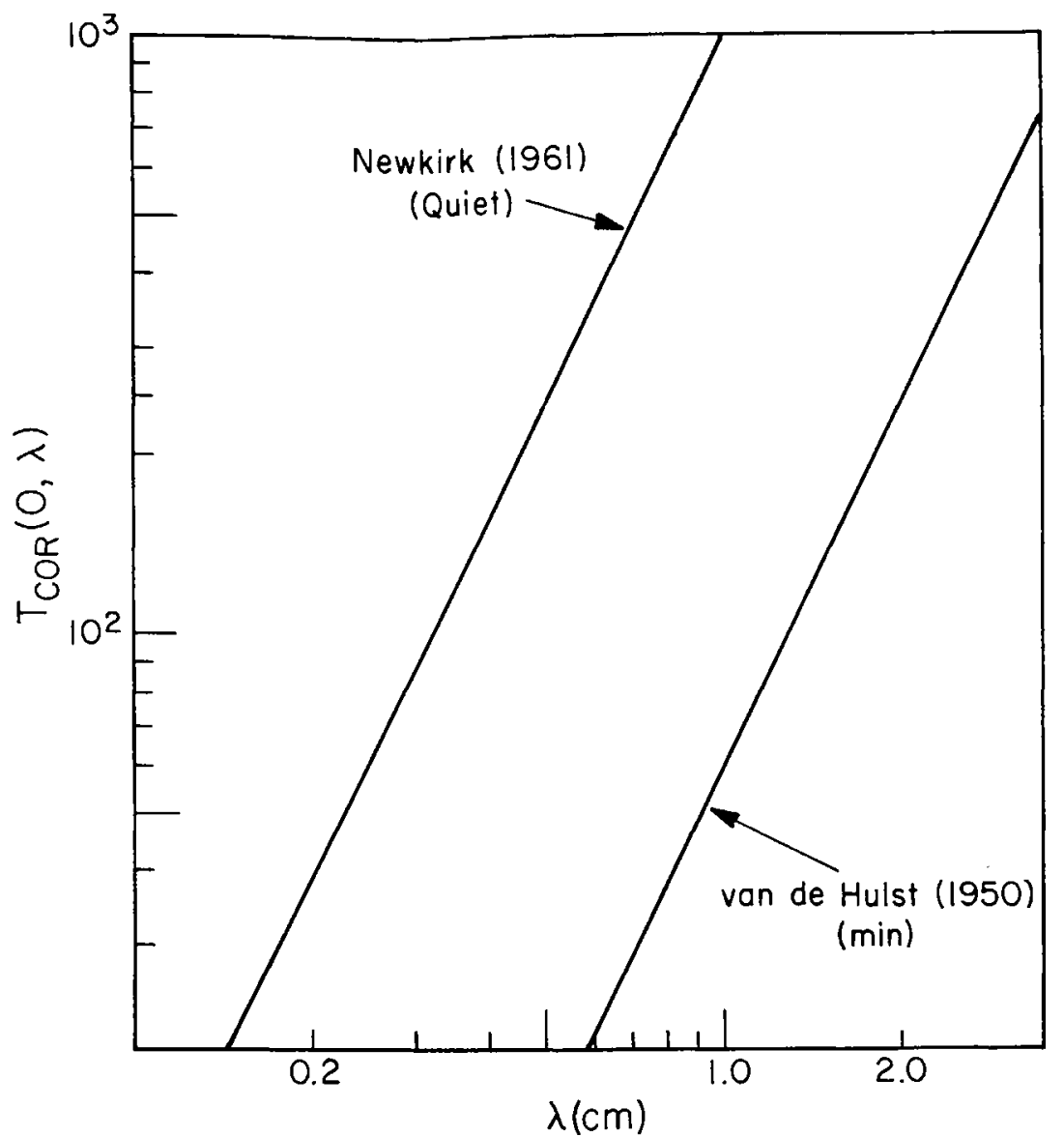

Fig. 5. Coronal contributions to the brightness temperature as a function of wavelength for the Newkirk (1961) and van de Hulst (1950) coronal models.

altitude were adjusted to give a flat brightness distribution at $8.6 \mathrm{~mm}$. A third component, identified with spicules, was added at a higher altitude to obtain the limb spike. The model required an extensive hot layer which would now be in conflict with the $2 \mathrm{~cm}$ observations (too hot and too much limb brightening) and which would also be in conflict with the extreme ultraviolet observations (Moriyama, 1961; Zirin and Dietz, 1963). Since the flat distributions at longer wavelength were not known at that time, Athay was only developing an inhomogeneous chromospheric model to fit the short wavelength data. It is clear that the same problem now has to be faced for a wide range of radio data, and a more general formulation of an inhomogeneous model is called for.

We visualize a model that is rough at all radio wavelengths. On the average (the average being over an antenna beam) such a surface would have the same brightness over a large range of aspect angles, and thus would postpone any limb brightening until much closer to the limb than on a smooth surface. We expect that the scale of roughness seen at a given radio wavelength is proportional to the scale height $H$ of the particular region responsible for most of the emission. Thus at centimeter wavelengths where the brightness temperatures are $0\left(10^{4}\right)$, the scale heights of the regions sampled are a few hundred kilometers, and there will be many rough elements, say $n$, within the antenna 
beam which averages over them. The fluctuations in the mean brightness temperature thus measured will be roughly $\Delta n / n \propto H \propto T$. At centimeter wavelengths we estimate $\Delta n / n \sim 10^{-3}$ to $10^{-2}$ which certainly is not in conflict with the observations. The scale of roughness increases with the temperature of the relevant region, and is reflected in the greater fluctuations in the brightness distribution observed at decimeter wavelengths.

The increasing scale of inhomogeneity with height is certainly a well-observed phenomenon. In the chromosphere considerable fine structure with scales of $1000 \mathrm{~km}$ or less is observed, while the corona is often observed to consist of a few large streamers which give the same brightness at various positions since we see as much of them straight on or sidewise.

\section{Acknowledgements}

We are very grateful to A. Tlamicha for sending to us the $2 \mathrm{~cm}$ data in advance of publication, and to A.T. Moffet for obtaining the $3 \mathrm{~cm}$ data at Goldstone. We thank $\mathrm{K}$. Chu for assistance with some of the model calculations.

Note added in manuscript: To obtain the brightness temperature distribution near the limb at millimeter wavelengths, we shall observe the sun during the partical eclipse of Sept. 11, 1969. We have carried out some preliminary observations at $9 \mathrm{~mm}$ with the N.R.A.O. 36-ft antenna at Kitt Peak and report some of the results here. To determine $T_{b}(0.9 \mathrm{~mm})$ we compared the flux of a quiet region in the sun with that of the center of the moon in its last quarter, and obtained a ratio of $31.2 \pm 0.8$. Using the lunar phase curve of Salomonovich and Losovskii (Soviet Astronomy-A.J. 6 (1963) 833) at $8 \mathrm{~mm}$, we obtain $T_{b}(0.9 \mathrm{~mm})=6900 \pm 500 \mathrm{~K}$ which falls near the lower bound of the swath in figure 1 . North-south scans showed that the brightness distribution was flat to at least $\varrho=0.7$. We could not of course obtain any detailed information on the distribution near the limb. While our observations did not indicate a large amount of limb brightening, they would be consistent with the results of Coates et al. (1958).

We are indebted to K. I. Kellermann for the use of some of his observing time, and to the staff of the observatory for assistance with the observations.

\section{References}

Allen, C. W.: 1963, Astrophysical Quantities, Oxford University Press, New York.

Alon, I., Arsac, J., and Steinberg, J. L.: 1953, Compt. Rend. 237, 300.

Athay, R. G.: 1959, Paris Symposium on Radio Astronomy.

Bonnett, R. M. and Blamont, J. E.: 1968, Solar Phys. 3, 64.

Buhl, D. and Tlamicha, A.: 1968, Astrophys. J. Letters 153, L189.

Coates, R. J.: 1958, Astrophys. J. 128, 83.

Coates, R. J., Gibson, J. E., and Hagen, J. P.: 1958, Astrophys. J. 128, 406.

Gingerich, O. and De Jager, C.: 1968, Solar Phys. 3, 5.

Kundu, M.: 1965, Solar Radio Astronomy, Interscience, New York.

Lena, P. J.: 1968, Solar Phys. 3, 28.

Little, A. G.: 1967, Proc. A.S.A. 1, 42. 
Moffet, A. T.: 1967, personal communication.

Moriyama, F.: 1961, Ann. Tokyo Astron. Obs. 7, 132.

Newkirk, G.: 1961, Astrophys. J. 133, 982.

Newstead, R.: 1969, Solar Phys. (in press).

Noyes, R. W. and Goldberg, L.: 1968, AAS Special Solar Mtg., Tucson, Ariz.

Noyes, R. W., Beckers, J. M., and Low, F. J.: 1968, Solar Phys. 3, 36.

Riddle, A. C.: 1969, Solar Phys. (in press).

Shimabukuro, F. I.: 1969, preprint.

Shimabukuro, F. I. and Stacey, J. M.: 1968, Astrophys. J. 152, 777.

Simon, M.: 1965, Astrophys. J. 141, 1513.

Swarup, G.: 1961, Rept. No. 13, Stanford Electronics Laboratories, Stanford, Calif.

Tlamicha, A.: 1968, personal communication.

Tousey, R.: 1967, Astrophys. J. 149, 239.

Vaiana, G. S., Reidy, W. P., Zehnpfennig, T., Van Speybroeck, L., Giaconni, R.: 1968, Science 161, 564.

Van de Hulst, H. C.: 1950, Bull. Astron. Inst. Netherlands 11, 135.

Zirin, H. and Dietz, R. D.: 1963, Astrophys. J. 138, 664. 